Nervenarzt 2009 $\cdot 80: 1341-1342$

DOI 10.1007/s00115-009-2883-1

(C) Springer Medizin Verlag 2009
M. Linden ${ }^{1} \cdot$ W. Maier ${ }^{2} \cdot$ H.-J. Möller ${ }^{3} \cdot$ H. Saß ${ }^{4}$

${ }^{1}$ Forschungsgruppe Psychosomatische Rehabilitation an der Charité Universitätsmedizin Berlin, Teltow/Berlin

${ }^{2}$ Klinik und Poliklinik für Psychiatrie und Psychotherapie, Bonn

${ }^{3}$ Psychiatrische Klinik der Ludwig-Maximilians-Universität, München

${ }^{4}$ Universitätsklinikum Aachen

\title{
Wissenschaft lebt von der Auseinandersetzung
}

\author{
Zur Debatte um die Evidenzbasierung \\ in der Psychotherapie
}

In der Rubrik Aktuelles aus Der Nervenarzt wurde ein Manuskript von Rief und Hofmann mit dem Titel „Die Psychoanalyse soll gerettet werden. Mit allen Mitteln?" publiziert [1]. Es handelte sich um die Kommentierung einer Arbeit von Leichsenring und Rabung im JAMA mit dem Titel „The effectiveness of long-term psychodynamic psychotherapy: a metaanalysis" [2]. Die Arbeit im JAMA hatte auch dort eine intensive Reaktion und Diskussion hervorgerufen mit Befürwortungen wie Kritik.

Die Rubrik Aktuelles in Der Nervenarzt hat zum Ziel, aktuelle wissenschaftliche Themen aufzugreifen und den Leser nicht nur in Form eines wissenschaftlichen Reviews zu informieren, sondern zugleich auch eine persönliche Einordnung und Bewertung durch die jeweiligen Autoren zu ermöglichen. Daher war es ein Anliegen der Herausgeber, der Diskussion der im JAMA aufgeworfenen Fragen auch in der deutschen psychiatrischen Fachzeitschrift Raum zu geben.

Die Kommentierung der Arbeit von Leichsenring und Rabung durch Rief und Hofmann hat nun wieder ein Reihe von sehr engagierten Leserbriefen und Stellungnahmen zur Folge gehabt. Die Herausgeber haben die Stellungnahmen ausgewählt, welche die Kritik an den Argumenten von Rief und Hofmann in die Form einer wissenschaftlichen Disputation gefasst haben. Sie haben zudem Leich- senring und Rabung gebeten, auch selbst eine Entgegnung zu schreiben. AbschlieBend erhielten Rief und Hofmann nochmals die Möglichkeit, auf die Argumente gegen ihre Argumente einzugehen.

Entstanden ist ein wissenschaftlicher Diskurs, der nach Auffassung der Herausgeber von Der Nervenarzt und der Rubrik Aktuelles durchaus außergewöhnlich ist und einer Fachzeitschrift gut ansteht. Wissenschaft lebt von der Auseinandersetzung, auch von der engagierten Auseinandersetzung. Die Herausgeber würden gerne in der Zukunft in ähnlicher Weise anderen derartigen offenen Positionsklärungen Raum geben.

\section{( Die Forderung nach Evidenzbasierung ist in Bereiche exekutiven Handelns eingedrungen}

Die Herausgeber haben die vorliegende Debatte aber auch unabhängig vom aktuellen Thema noch aus einer grundsätzlichen Überlegung heraus gerne publiziert. Die Diskussion rührt an einen Kernpunkt des aktuellen Wissenschaftsverständnisses. Mit der wachsenden Bedeutung einer ,evidenzbasierten Medizin“ stellt sich zunehmend schärfer das Grundlagenproblem, was eigentlich ,evidenzbasiert" ist. Solange dies eine akademische Frage ist, kann man problemlos mit einer gewissen Unschärfe leben und jedem Wissenschaftler seine Lieblingsdefinition bzw. -auffassung zubilligen. Die Zeiten haben sich jedoch geändert. Die Forderung nach Evidenzbasierung ist schon lange kein wissenschaftliches Thema mehr, sondern zu einem regulativen Mechanismus geworden. Der wissenschaftliche Beirat Psychotherapie, die Arzneimittelzulassungsbehörden, der Gemeinsame Bundesausschuss der Ärzte und Krankenkassen, das Institut für Qualität und Wirtschaftlichkeit im Gesundheitswesen IQWUiG, Kostenträger im Gesundheitssystem und die Politik betrachten die Evidenzbasierung als feste Größe und begründen damit einschneidende regulative Eingriffe in das Gesundheitswesen und die tägliche ärztliche Praxis. Die ursprünglich wissenschaftliche Problemstellung ist somit in Bereiche exekutiven Handelns eingedrungen, und damit wird es ernst. Deshalb ist die Wissenschaft nun wieder in besonderer Weise herausgefordert, die Probleme mit dem Konzept der Evidenzbasierung aufzuzeigen, die verschiedenen wissenschaftlichen Arbeitsansätze und wissenschaftlichen Ideologien herauszuarbeiten, gegeneinanderzustellen und abzuwägen.

Hierzu kann der vorliegende Diskurs um die Evidenzbasierung der Psychoanalyse einen hervorragenden Beitrag leisten. Die Autoren der einzelnen Beiträge sind für das, was sie geschrieben haben, in Inhalt und Form selbst verantwortlich, 
die Herausgeber haben nicht korrigierend eingegriffen, sie stellen die Plattform zur Auseinandersetzung zur Verfügung. Nun ist es am Leser zu entscheiden, welche Argumente überzeugend sind.

\section{Korrespondenzadressen}

\section{Prof. Dr. M. Linden}

Forschungsgruppe Psychosomatische Rehabilitation an der Charité Universitätsmedizin Berlin Lichterfelder Allee 55, 14513 Teltow/Berlin michael.linden@charite.de

\section{Prof. Dr. H. Saß}

Universitätsklinikum Aachen

Pauwelstraße 30, 52057 Aachen

hsass@ukaachen.de

\section{Literatur}

1. RiefW, Hofmann S (2009) Die Psychoanalyse soll gerettet werden. Mit allen Mitteln? Nervenarzt 80:593-597

2. Leichsenring F, Rabung $S$ (2008) The effectiveness of long-term psychodynamic psychotherapy: a meta-analysis. JAMA 300:1551-1565

\section{Meinolf Bachmann, Andrada El-Akhras Lust auf Abstinenz}

Ein Therapiemanual bei Alkohol-, Medikamenten- und Drogenabhängigkeit Heidelberg: Springer Verlag 2009, 180 S., (ISBN 978-3-540-89225-0), Softcover, 39.95 EUR

Der Titel "Lust auf Abstinenz" verblüfft zunächst. Bisher schon war es nicht einfach, Begriffe wie „zufriedene Abstinenz" oder noch niedrigschwelliger "suchtmittelfreies Leben" o. ä. zu installieren. Doch diese für einen Suchtkranken scheinbar paradoxe Aufforderung könnte ohne Zweifel motivierend wirken: Denn Suchtmittelabstinenz ist für inn in Wirklichkeit kein Verzicht, sondern Befreiung. Es kann keiner mehr bestreiten, dass bei den meisten Suchtformen die konsequente Abstinenz der einfachste und sicherste Weg zu einem besseren Leben ist.

Das Werk wird auf dem Umschlag als "Therapiemanual" bezeichnet, also zu Händen der Therapeuten gedacht. Dies entspricht auch der Verlagsankündigung, nach der die in diesem "Arbeitsbuch" zusammengestellten „Arbeitsmaterialien" den Behandlungsablauf strukturieren sollen und für "Klinische Psychologen, Psychiater, Psychotherapeuten " bestimmt ist. Doch fällt auf, dass keine DVD zum Ausdrucken der Arbeitsmaterialien beigefügt ist, wie es sich bei Therapiemanualen in letzter Zeit bewährt hat.

Tatsächlich kann das Werk wesentlich mehr sein: Schon auf Seite 2 heißt es „in diesem Arbeitsheft" und "Therapiebegleitheft". Dies bedeutet, dass jeder Patient einer stationären, tagesklinischen oder ambulanten Suchttherapie sich dieses Werk anschafft (es kostet ja nur so viel wie etwa der Wochenbedarf an Alkohol), während der Therapie damit arbeitet und nach der Therapie immer wieder mal nachsehen kann, was er selbst damals in dieses Therapiebegleit-Buch bzw. Arbeitsheft geschrieben hat, was die Wirkungsdauer der Therapie ohne Zweifel verlängert.

Das Manual hat 10 Kapitel. Das 1. Kapitel enthält eine wohl auch für uninformierte Laien gut lesbare Einführung in unser derzeitiges Wissen über die Suchtkrankheit. Die Kapitel 2, 3 und 9 befassen sich mit dem Therapieablauf: die erste Zeit des Entzugs und der Entwöhnung, Prozesse im Gehirn/Belohnungszentrum und Suchtgedächtnis, Therapieplanung, Ursachenforschung und Veränderungsschritte und ähnlichem. Die Kapitel 4 bis 8 haben als Thema:„Beziehungen und soziale Kompetenzen", Gefühle zeigen - Gefühlskiste", „Geld zum Thema machen“", Rückfallverhütung" und "Alternativen zum Suchtverhalten: Struktur und Aktivitätsplan“. Das letzte Kapitel enthält ein kurzes und prägnantes Schlusswort zum Weg in eine zufriedene Abstinenz. Zudem bietet das Therapiemanual Vorgaben für Rollenspiele, bspw. Rückfallvorwurf von der Partnerin. In jedem dieser praxisorientierten Kapitel finden sich eine Einführung und mehrere Arbeits- und Infoblätter. Ein Arbeitsblatt enthält meist 20 bis 50 Fragen oder Aussagen mit vorgegebenen Antwortmöglichkeiten (", trifft gar nicht zu“ usw. oder 0 bis 6) und Raum für persönliche Notizen. Diese Arbeitsblätter basieren merklich auf langjährigen Erfahrungen in der stationären Sucht-Psychotherapie und wurden immer wieder überprüft und verbessert. Als Beispiel das letzte Arbeitsblatt: die Überschrift "Wöchentliches Zufriedenheitsbarometer", 35 Aussagen, je 5 Antwortmöglichkeiten ${ }_{1}, 0=$ überhaupt nicht verwirklicht " ${ }^{\prime \prime}{ }_{11} 4$ $=$ sehr gut verwirklicht"). Es wird angeregt, diese Arbeitsblätter zunächst in Kleingruppen zu diskutieren.

Es liegt nahe, dass auf diese Weise Selbstexploration, Einstellungswandel etc. intensiv angeregt und gefördert werden. Dies ist auch dringend erforderlich angesichts der gegenüber den „Halbjahreskuren“ ("Kurlaub“) der 1970er und 1980er Jahre erheblich verkürzten Zeit stationärer Suchttherapie, die intensiv genutzt werden muss. Dabei ging nichts verloren, was schon früher sich als therapeutisch wertvoll erwiesen hat, bspw. der Terminus "Wachsamkeit", den Bachmann in den 80er Jahren eingeführt hat.

Für die 2. Auflage wäre zu erwägen, eine DVD mit den Arbeitsblättern etc. beizufügen, um die Handlichkeit für die Suchttherapeuten zu verbessern. Zu erwägen wäre auch, zur Ergänzung der kognitiven Therapieaspekte ein weiteres Kapitel mit dem Thema „Selbstdiagnose“ bzw. „Selbstbetrugs-Taktiken“ (,Ich bin Alkoholiker") hinzu zu geben.

Für jede suchtpsychotherapeutische stationäre, ambulante oder tagesklinische Einrichtung für Substanzabhängige, aber auch für Verhaltenssüchtige kann dieses Therapiemanual wertvoll, zumindest anregend und bereichernd sein.

Dr. Bert Kellermann (Hamburg) 